\title{
Adaptação transcultural do Late-life Function and Disability Instrument para o Português Brasileiro
}

\section{Cross-cultural adaptation of Late-life Function and Disability Instrument into Brazilian Portuguese}

\author{
Rosé Colom Toldrá1, Ana Cristina Fagundes Souto², Marina \\ Picazzio Perez Batista ${ }^{3}$, Maria Helena Morgani de Almeida ${ }^{4}$
}

TOLDRÁ, R. C.; SOUTO, A. C. F.; BATISTA, M. P. P.; ALMEIDA, M. H. M. Adaptação transcultural do Late-life Function and Disability Instrument para o Português Brasileiro. Rev. Ter. Ocup. Univ. São Paulo, v. 23, n. 1, p. 52-61, jan./abr. 2012.

RESUMO: O presente artigo apresenta o processo de adaptação transcultural para o Português Brasileiro do "Late-life Function Disability Instrument - LLFDI", destinado a avaliar o grau de independência funcional de pessoas acima de 60 anos que moram na comunidade e captar mudanças no nível funcional e no grau de incapacidade. O instrumento compreende dois componentes: Função e Incapacidade. O componente de Função avalia o grau de dificuldade para a realização das atividades e o de Incapacidade afere duas dimensões: a freqüência de realização das atividades e o grau de limitações. O processo de adaptação transcultural compreendeu tradução, retrotradução, análise por Comitê de Especialistas e pré-teste conduzido com 47 sujeitos. O “Instrumento de Avaliação da Função e Incapacidade para a População Idosa - LLFDI-Br” produzido foi plenamente compreendido, podendo se constituir como uma importante ferramenta de avaliação para esta população. Novos estudos deverão ser conduzidos para avaliação de suas propriedades psicométricas.

DESCRITORES: Envelhecimento; Idoso; Avaliação/métodos; Tradução; Adaptação; Funcionalidade; Incapacidade; Classificação Internacional de Funcionalidade, Incapacidade e Saúde.

1. Prof ${ }^{a} \operatorname{Dr}^{\mathrm{a}}$ do Curso de Terapia Ocupacional do Departamento de Fisioterapia, Fonoaudiologia e Terapia Ocupacional - Faculdade de Medicina Universidade de São Paulo.

2. Terapeuta Ocupacional do Curso de Terapia Ocupacional do Departamento de Fisioterapia, Fonoaudiologia e Terapia Ocupacional - Faculdade de Medicina Universidade de São Paulo.

3. Terapeuta Ocupacional do Curso de Terapia Ocupacional do Departamento de Fisioterapia, Fonoaudiologia e Terapia Ocupacional - Faculdade de Medicina Universidade de São Paulo.

4. Prof $^{a} \operatorname{Dr}^{\mathrm{a}}$ do Curso de Terapia Ocupacional do Departamento de Fisioterapia, Fonoaudiologia e Terapia Ocupacional - Faculdade de Medicina Universidade de São Paulo.

Endereço para correspondência: Rosé Colom Toldrá. Rua Cipotânea, 51 - Cidade Universitária - S.P. - São Paulo, SP. E-mail: rosetoldra@usp.br 
TOLDRÁ, R. C. et al. Adaptação transcultural do Late-life. Rev. Ter. Ocup. Univ. São Paulo, v. 23, n. 1, p. 52-61, jan./abr. 2012.

\section{INTRODUÇÃO}

$\mathrm{O}$ envelhecimento populacional é uma realidade mundial. O Brasil está entre os países com ritmo mais acentuado de envelhecimento e as projeções indicam que em 2025 haverá mais de 50 adultos com 65 anos ou mais para cada conjunto de 100 jovens menores de 16 anos, e em 2045 o número de pessoas idosas ultrapassará o de crianças (WONG; CARVALHO, 2006).

Aindependência funcional éconsiderada importante medida para promoção de saúde no envelhecimento. O grau de independência funcional está intrinsecamente relacionado ao conceito de capacidade funcional que compreende a capacidade da pessoa em manter suas habilidades mentais e físicas demandadas para uma vida independente e autônoma (BRASIL, 1999, 2006).

Ao considerar a questão da incapacidade funcional, as projeções indicam um contingente de 6,7 milhões de idosos nesta condição em 2025, chegando aos 12 milhões no ano de 2050 (WONG; CARVALHO, 2006). Estudos conduzidos no Brasil indicam altas prevalências de incapacidades para, pelo menos, uma das atividades diárias, variando de $28 \%$ a 61\% (LITVOC; DERNTL, 2002).

A detecção precoce e o tratamento de limitações funcionais, que potencialmente causam dependência aos idosos, são uma das atribuições dos profissionais da área gerontológica (BRASIL, 1999). A Política Nacional de Saúde da Pessoa Idosa reconhece a relevância da avaliação da capacidade funcional e de intervenções que visem à prevenção e recuperação do declínio funcional e, para tanto, considera que a aplicação de instrumentos de avaliação da funcionalidade se apresenta como uma das estratégias para detecção precoce de possíveis comprometimentos (BRASIL, 2006).

Neste sentido necessária a utilização de instrumentos confiáveis para a realização de avaliação geriátrica eficiente e completa, que compreenda os distintos aspectos relacionados às funções necessárias à vida diária do idoso a fim de se atuar o mais preventivamente possível sobre os problemas de saúde e orientar serviços de apoio (PAIXÃO Jr; REINCHENHEIM, 2005).

A aplicação de instrumentos de avaliação contribui para o planejamento de recursos humanos, ambientais, materiais e oferece uma base objetiva para a intervenção. Sua reaplicação, em intervalos regulares, estima mudanças no tempo, permite avaliar resultados do tratamento e sua reformulação (LAWTON; BRODY, 1969). Ainda, ao focalizarem o comportamento concreto em uma linguagem cotidiana, as escalas permitem sua utilização e comunicação entre diferentes profissionais e serviços, além da avaliação das consequências de hospitalizações, declínio funcional, tempo de internação e institucionalização (LAWTON; BRODY, 1969; MELLO; MANCINI, 2007).

Embora sejam úteis para aplicação em diferentes condições, reconhece-se que há poucos instrumentos validados para o português. A carência de textos neste idioma que orientem o processo de adaptação e validação de instrumentos em diferentes áreas da saúde e que contemplem o contexto cultural e estilo de vida da população alvo indica a importância desta área de estudo (REICHENHEIM; MORAES, 2007; MELLO; MANCINI, 2007).

Tal fator associado às possibilidades já citadas quanto ao uso de instrumentos de avaliação e escalas, bem como o aumento de idosos, sinalizam a importância de ferramentas específicas para avaliação deste segmento populacional.

Este artigo apresenta o processo de adaptação transcultural do "Late-life Function and Disability Instrument - LLFDI” enquanto um instrumento concebido para uso específico com idosos.

\section{O LATE-LIFE FUNCTION AND DISABILITY INSTRUMENT (LLFDI)}

O LLFDI foi desenvolvido por JETTE et al. em 2002 nos Estados Unidos, com o objetivo de avaliar de forma abrangente, por meio de entrevistas, a função e a incapacidade em idosos que moram na comunidade e ser sensível para captar mudanças experimentadas por estes indivíduos nestes dois aspectos.

Este Instrumento foi concebido a partir do modelo de Incapacidade proposto por Nagi (1976, apud JETTE et al., 2002), que compreende as limitações funcionais como limitações nas habilidades das pessoas em realizarem distintas tarefas físicas, e incapacidade como o impacto dessas limitações na participação das pessoas em tarefas socialmente esperadas em um contexto sociocultural e ambiental específico. Foi desenvolvido a partir da constatação da fragilidade dos instrumentos existentes para detectar mudanças ocorridas em resposta às intervenções.

O processo de construção do LLFDI envolveu a revisão dos instrumentos existentes, submissão a um comitê de especialistas na área da reabilitação e envelhecimento e grupos focais com idosos que residem na comunidade, resultando em dois componentes: "Function" e "Disability", os quais podem ser aplicados em conjunto ou separadamente.

O componente "Disability" é composto por 16 itens e mede duas dimensões: "Frequency" que se refere 
à frequência da participação nas atividades cotidianas e "Limitation" que corresponde à capacidade percebida pelo sujeito para realizá-las, considerando a influência dos fatores pessoais (como saúde, energia física ou mental) e fatores ambientais (como transporte, acessibilidade e questões socioeconômicas). Essa forma de organização foi adotada considerando que as intervenções podem afetar de forma diferente cada uma dessas dimensões.

Na dimensão "Frequency" são identificados dois domínios: "Social Role Domain", relativo às atividades sociais e comunitárias e "Personal Role Domain" relativo às atividades pessoais. Na dimensão "Limitation" são identificados 2 domínios: "Instrumental Role Domain", que abrange atividades em casa e na comunidade e o "Management Role Domain”, referente à organização ou administração de tarefas sociais que envolvem primordialmente habilidades cognitivas.

O componente "Function" é composto por 32 itens que se referem à habilidade para realização de atividades específicas que requerem atos motores amplos e finos. Seus itens avaliam 3 domínios de função: "Advanced Lower Extremity Functioning" que se refere às funções de membros inferiores que exigem um alto nível de habilidade física e resistência; "Basic Lower Extremity Functioning" relativo às atividades que envolvem primordialmente funções básicas dos membros inferiores, tais como: permanecer em pé, inclinar-se e caminhar, e o "Upper Extremity Functioning”, que corresponde às atividades que utilizam os membros superiores.

O domínio “Management Role Domain”, voltado principalmente ao desempenho cognitivo, assemelha-se ao que alguns profissionais chamam de Atividades Avançadas de Vida Diária (AAVD) (JETTE et al. em 2002). As dificuldades neste nível de atividades podem não indicar perda funcional atual importante, mas riscos de perdas futuras (PAIXÃO Jr; REINCHENHEIM, 2005).

Diversos estudos levantados por Dias et al. (2011) apontam que o monitoramento das AAVDs pode detectar mudanças na capacidade funcional ao longo do tempo.

A funcionalidade, em geral, é avaliada por dois tipos de medidas: as baseadas em desempenho e as autodeclarativas. Diversos autores questionam a utilização das medidas auto-declarativas por serem consideradas limitadas quanto à sensibilidade às mudanças, sua reprodutibilidade e incapacidade de captar uma ampla gama de formas de funcionamento (LINN; LINN, 1980; REUBEN, 1995; VERBRUGGE; JETTE, 1994 apud SAYERS et al., 2004).

Visando comprovar as propriedades avaliativas do LLFDI, foi realizada a validação concorrente pela comparação com medidas de desempenho, no caso, "400Meter Self-Paced Walk" e o "Short Physical Performance Battery”, em uma amostra de 101 indivíduos com idades de 75 a 90 anos, que resultou em forte correlação na dimensão "Advanced Lower Extremity Function” e correlação significativa na dimensão "Limitation" do LLFDI. A falta de associação entre as medidas de comparação e a dimensão "Frequency" do LLFDI parece reforçar a idéia de que a incapacidade física é influenciada não somente por aspectos físicos, mas também por fatores ambientais e comportamentais, mas ainda, que para cada nível de funcionalidade, pode haver mudanças na freqüência de realização, não somente no grau de limitação. (SAYERS et al., 2004).

Deste modo a correlação entre as medidas de desempenho acima citadas e os domínios do LLFDI reafirmam sua utilidade enquanto instrumento autodeclarativo para a avaliação funcional de idosos.

O LLFDI foi validado por meio de aplicação em uma amostra de 150 adultos com 60 anos ou mais, morando na comunidade, tendo sido excluídos aqueles com erros em cinco ou mais itens do "Short Portable Mental Status Questionnaire”, classificados com distúrbio moderado a severo, além de hospitalização nos últimos 6 meses ou incapacidade de se levantar da cama. Os sujeitos foram divididos em 4 estratos de acordo com a função física, de forma a distribuí-los em níveis distintos de capacidade funcional. Os elegíveis foram entrevistados por profissional treinado e a média de aplicação dos dois componentes foi de 25 minutos. Uma sub-amostra de 15 pessoas foi reentrevistada no período de 1 a 3 semanas após a entrevista inicial, para determinação da confiabilidade teste-reteste. As correlações intraclasse indicaram uma boa confiabilidade teste-reteste e o coeficiente alfa de Cronbach refletiu uma consistência interna de aceitável a excelente (alfa de Cronbach 63-.92) (McAULEY et al., 2005).

Em 2005, McAuley et al. desenvolveram a versão abreviada, denominada "SF-LLFDI", composta por 8 itens na escala "Disability" e 15 na escala "Function". Esta versão foi adaptada para o Alemão (DENKINGER et al., 2009), Espanhol (ABIZANDA et al., 2011) e Coreano (SHIN et al., 2008). A versão completa do instrumento foi adaptada apenas para o Hebraico (MELZER et al., 2007).

\section{MATERIAIS E MÉTODOS}

O processo de adaptação transcultural para o Português Brasileiro do "Late-life Functional Disability Instrument - LLFDI" inscreve-se no projeto intitulado "Envelhecer Mantendo Funções: idosos no ano de 2020" 
TOLDRÁ, R. C. et al. Adaptação transcultural do Late-life. Rev. Ter. Ocup. Univ. São Paulo, v. 23, n. 1, p. 52-61, jan./abr. 2012.

(MANSUR et al., 2009), que tem dentre seus objetivos a adaptação e produção de instrumentos para diagnóstico nas áreas de comunicação, movimento e ocupação. A pesquisa foi aprovada pelo Comitê de Ética de Pesquisa do Hospital Universitário da USP sob o $n^{\circ} 1005 / 10$. O processo de adaptação transcultural do LLFDI para o contexto sociocultural brasileiro teve início em janeiro de 2011, após a obtenção de autorização do autor principal, Allan Jette, e finalização em fevereiro de 2012.

O protocolo para adaptação transcultural do LLFDI foi elaborado e desenvolvido de acordo com as etapas propostas pela literatura: tradução do instrumento, síntese das traduções, retrotradução, comitê de especialistas e pré-teste (GUILLEMIN et al., 2003; REICHENHEIM; MORAES, 2007; BEATON et al., 2007).

Os participantes assinaram Termo de Consentimento Livre e Esclarecido conforme Resolução 196/96 do Conselho Nacional de Saúde.

\section{RESULTADOS E DISCUSSÃO}

O processo de adaptação transcultural do LLFDI para o português brasileiro consistiu nas etapas sintetizadas na Figura 1.

A tradução foi realizada, de forma independente, por dois professores de língua inglesa, brasileiros, cientes dos objetivos do trabalho de adaptação transcultural e da finalidade do instrumento, tendo sido obtidas as "Tradução 1" e "Tradução 2". Estas traduções foram analisadas por um subcomitê composto por dois professores e três terapeutas ocupacionais da área de envelhecimento e funcionalidade, produzindo uma única versão: a "Tradução Consensuada".

Esta foi submetida à retrotradução por dois tradutores norteamericanos, cuja língua materna é o inglês, e que desconheciam os objetivos do trabalho.

Considerando as diferenças encontradas nas retrotraduções, optou-se pela constituição de um Comitê de Especialistas, composto por nove profissionais: um tradutor, dois retrotradutores, um terapeuta ocupacional com experiência em adaptação de instrumentos na área de gerontologia, um fisioterapeuta com experiência clínica em gerontologia e quatro terapeutas ocupacionais, executoras do projeto, sendo dois coordenadores e dois relatores do processo.

Todos os membros do comitê receberam, com antecedência, orientações quanto ao seu objetivo e um quadro comparativo contendo todas as versões: original, tradução consensuada e retrotradução 1 e 2 dos itens dos componentes, orientações e esquemas visuais. Este material foi previamente preparado e encaminhado ao comitê com base em consulta feita a um dos autores do "Recommendations for the Cross-Cultural Adaptation of the DASH \& Quick DASH Outcome Measures" (BEATON et al., 2007).

O comitê analisou as versões produzidas e sugeriu alterações que levaram em consideração a equivalência semântica, idiomática e conceitual. A partir destas alterações foi produzida a versão $n^{\circ} 1$ em português, utilizada na aplicação no pré-teste 1 .

O pré-teste 1 foi conduzido no período de julho a setembro de 2011 no Hospital Universitário da USP/SP com uma amostra de 30 pessoas, residentes em São Paulo e municípios adjacentes, participantes do "Projeto Envelhecer Mantendo Funções”.

Figura 1. Etapas do processo de adaptação transcultural do LLFDI para o português brasileiro

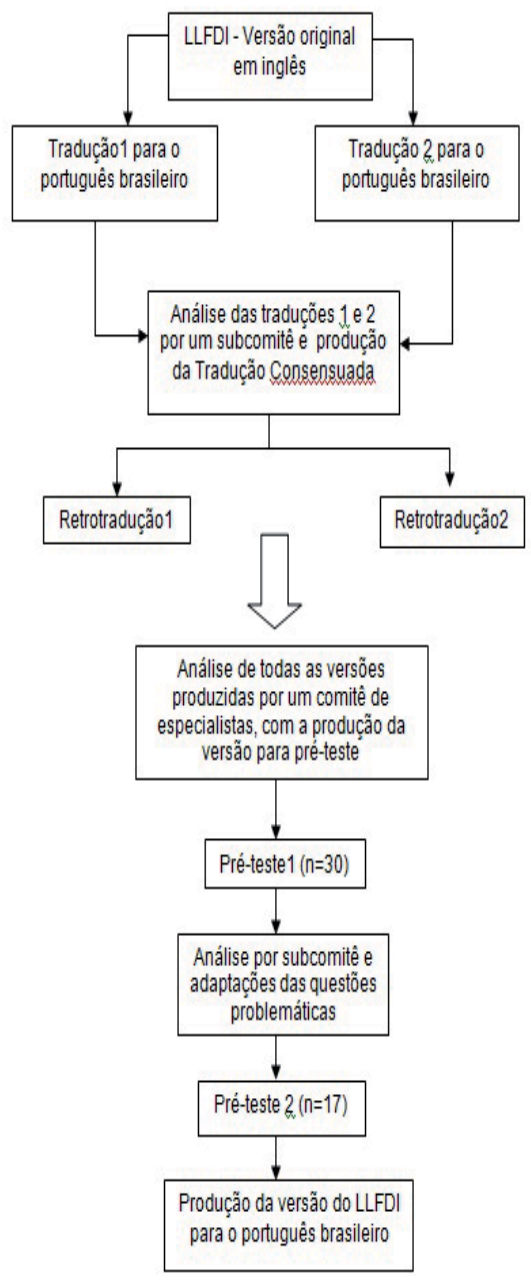


TOLDRÁ, R. C. et al. Adaptação transcultural do Late-life. Rev. Ter. Ocup. Univ. São Paulo, v. 23, n. 1, p. 52-61, jan./abr. 2012.

Quanto ao perfil dos sujeitos do pré-teste 1, 17 (57\%) eram do sexo feminino e em relação à faixa etária 3 (10\%) tinham entre 50 a 59 anos; 18 (60\%) entre 60 a 69 anos e 9 (30\%) com 70 anos ou mais.

As entrevistas ocorreram em uma sala fechada, com boa iluminação e sem interferências. A média de tempo para aplicação dos dois componentes do instrumento, com apoio dos respectivos esquemas visuais, foi de 28 minutos, tempo esse muito próximo daquele despendido na aplicação da versão original pelo autor, que foi de 25 minutos (JETTE et al., 2002).

Os entrevistados foram orientados a interromper e questionar o entrevistador quando houvesse necessidade de repetição ou explicações sobre o conteúdo das perguntas e para darem sugestões sobre a melhor formulação, sendo as mesmas consideradas na análise das ocorrências.

A literatura que se refere à adaptação de instrumentos propõe um parâmetro para a quantidade de ocorrência das dúvidas de interpretação a ser considerado para a realização de alterações na tradução. Este parâmetro varia de maior ou igual a 10\% (REICHENHEIM; MORAES, 2007) até maior ou igual a 15\% (CICONELLI et al., 1999). Neste estudo considerou-se para análise a ocorrência de dúvidas em, no mínimo, $10 \%$ da amostra.

Quanto às perguntas-chave dos componentes, a questão IA (dimensão Frequência do Componente Incapacidade) suscitou dúvidas em 10\% (3) dos participantes, enquanto a questão IB (dimensão Limitação do Componente Incapacidade) suscitou dúvidas em 57\% (17) dos participantes. Quanto à questão F (Componente Função), não houve dificuldades de compreensão.

Foram detectadas dúvidas em 11 itens no préteste 1, sendo cinco no componente Função $(F)$ e seis no componente Incapacidade (D) conforme Tabela 1.

A partir das evidências encontradas no pré-teste 1 foram realizados ajustes semânticos por um subcomitê de especialistas, dando origem à versão 2 , aplicada em novo pré-teste a uma amostra de 17 sujeitos, não participantes do pré-teste 1.

Esta amostra foi composta por 82\% de mulheres com média de 65,1 anos de idade.

Em relação à versão 2 foram reaplicados todos os itens do componente "Incapacidade", dada a reformulação da questão central e apenas aqueles do componente "Função" que sofreram ajustes a partir do primeiro préteste. Como recomendado pelo autor do LLDFI, a aplicação do instrumento nos pré-testes (1 e 2) foi desenvolvida na forma de entrevista, por um profissional da área de terapia ocupacional.
Tabela1. Itens do instrumento com índice de ocorrência de dúvidas maior ou igual a $10 \%$

\begin{tabular}{l|l|l}
\hline Itens & $\begin{array}{l}\text { Número absoluto de } \\
\text { ocorrências }\end{array}$ & \% de ocorrências \\
\hline D.6 & 6 & 20,0 \\
\hline D.2 & 4 & 13,3 \\
\hline D.3 & 4 & 13,3 \\
\hline D.10 & 4 & 13,3 \\
\hline D.1 & 3 & 10,0 \\
\hline D.7 & 3 & 10,0 \\
\hline F.26 & 6 & 20,0 \\
\hline F.15 & 5 & 16,7 \\
\hline F.13 & 4 & 13,3 \\
\hline F.1 & 3 & 10,0 \\
\hline F.24 & 3 & 10,0 \\
\hline
\end{tabular}

O processo de adaptação transcultural do LLDFI levou em consideração o instrumento como um todo, ou seja, as instruções, os itens do questionário de ambos os componentes (Função e Incapacidade) e as escalas visuais. Buscou-se manter uma tradução a mais fiel possível, com equivalência para o idioma (português brasileiro). Esse processo compreendeu nova redação de alguns itens com o cuidado de preservar seu significado, substituição de termos não pertinentes à nossa cultura por outros, mas resguardando o conceito original. Desta forma objetivou-se atender a especificidade dos itens, alterando o menos possível a estrutura e as propriedades de avaliação das questões.

$\mathrm{O}$ autor do instrumento foi consultado quanto aos vários aspectos considerados durante todo o processo de adaptação, dos quais se destacam alguns exemplos. $\mathrm{O}$ primeiro refere-se ao nome do instrumento, mais especificamente ao termo "late-life", que caracteriza uma etapa da vida, o qual foi traduzido por "população idosa”, por designar o grupo populacional ao qual o instrumento se destina. Assim, o título da versão brasileira foi traduzido por: "LLFDI-Br - Instrumento de Avaliação da Função e Incapacidade para a População Idosa”.

Por se tratar de um instrumento para idosos foi incluído no questionário o tratamento "Sr." e Sr(a)" no início de cada questão, por ser respeitoso e comumente utilizado na interação com essa população no Brasil.

No pré-teste 1 , no componente incapacidade, a pergunta IA “Com que freqüência o Sr.(a)...?”, embora tenha sido compreendida pelos participantes, suscitou a necessidade de delimitar o tempo, pois o termo "neste 
momento”, contido nas instruções, parece não limitar claramente a temporalidade. Para tanto foi oferecida como orientação a utilização de “no último mês”, tendo em vista que instrumentos destinados à investigação de aspectos do momento atual como o SF-36 (CICONELLI et al, 1999) utilizam este período como critério de orientação. Tal alteração foi incorporada à versão final das instruções do instrumento.

Os participantes do pré-teste solicitaram esclarecimentos sobre como responderiam no caso de atividades que nunca realizam. Optou-se nesse caso por repetir as instruções que constam no componente função, ou seja, "posso inclusive dar exemplos de algumas atividades que o(a) Sr(a) de fato nunca faz.”

Referente ao componente Incapacidade, 57\% dos entrevistados tiveram dúvidas na pergunta IB "Até que ponto o(a) Sr (a) se sente limitado para...?” no pré-teste 1. Os entrevistados tenderam a confundir limitação com freqüência de realização, gostar ou não de determinada atividade e, ainda, ter ou não condição pessoal de realizála. Para o pré-teste 2 a pergunta foi reformulada: “Até que ponto o $\operatorname{Sr}(\mathrm{a})$. sente que há limitações para...?”. A substituição de "limitado" por "limitações” foi adotada para evitar a compreensão equivocada de que seria apenas um atributo do sujeito. Assim, para esclarecer a natureza complexa da relação entre sujeito e o ambiente, o entrevistador deverá reforçar as instruções, repetindo que as limitações para realizar as atividades podem ser de ordem física, mental, emocional, social, econômica e/ou ambiental. Com esta formulação a questão foi compreendida no pré-teste 2.

Para garantia da equivalência semântica, idiomática e cultural, alguns itens traduzidos foram modificados. $\mathrm{Na}$ Tabela 2 são apresentados exemplos de alterações realizadas no Componente Incapacidade, ocorrida no processo de adaptação.

Tabela 2. Exemplos de adaptações realizadas no Componente Incapacidade do LLFDI-Br

\begin{tabular}{|c|c|c|c|}
\hline Item & Original & Versão produzida & Observações \\
\hline D3 & $\begin{array}{l}\text { Provide (Providing) care or assistance to } \\
\text { others. This may include providing per- } \\
\text { sonal care, transportation, and running } \\
\text { errands for family members or friends. }\end{array}$ & $\begin{array}{l}\text { Cuida ou presta assistência a outras pessoas. } \\
\text { Isso pode incluir: prover cuidados pessoais, } \\
\text { transporte, fazer favores para membros da famí- } \\
\text { lia ou amigos. }\end{array}$ & $\begin{array}{l}\text { “Fazer tarefas para membros da família ou } \\
\text { amigos” foi alterada para "fazer favores”, } \\
\text { pois houve compreensão de que se tratava de } \\
\text { algum tipo de trabalho voluntário. }\end{array}$ \\
\hline D5 & $\begin{array}{l}\text { Work (Working) at a volunteer job out- } \\
\text { side your home. }\end{array}$ & $\begin{array}{l}\text { Assume (assumir) trabalhos voluntários fora } \\
\text { de casa. }\end{array}$ & $\begin{array}{l}\text { O termo “assume” foi adotado, pois enfatiza } \\
\text { a relação de compromisso, já que houve a } \\
\text { compreensão de que qualquer tarefa não } \\
\text { cobrada seria considerada voluntária. }\end{array}$ \\
\hline D6 & $\begin{array}{l}\text { Take (taking) part in active recreation. } \\
\text { This may include bowling, golf, tennis, } \\
\text { hiking, jogging, or swimming. }\end{array}$ & $\begin{array}{l}\text { Faz (fazer) atividades de lazer ativo. Isso pode } \\
\text { incluir: boliche, bocha, futebol/pelada, andar, } \\
\text { passear com cachorro, caminhada, dançar. }\end{array}$ & $\begin{array}{l}\text { Foi utilizado o termo “lazer”, pois na nossa } \\
\text { cultura “recreação” é compreendida como } \\
\text { atividade da infância. Os exemplos foram } \\
\text { alterados para diferenciar do item D10, } \\
\text { que trata de atividades de cunho esportivo, } \\
\text { enquanto o item D6 se refere às atividades } \\
\text { com potencial socializante e físico. }\end{array}$ \\
\hline D7 & $\begin{array}{l}\text { Take (taking) care of household business } \\
\text { and finances. This may include managing } \\
\text { and taking responsibility for your money, } \\
\text { paying bills, dealing with a landlord or } \\
\text { tenants, dealing with utility companies or } \\
\text { governmental agencies. }\end{array}$ & $\begin{array}{l}\text { Cuida (cuidar) da administração da casa e das } \\
\text { finanças. Isso pode incluir: saber quanto ganha, } \\
\text { como está sua conta bancária e se responsabili- } \\
\text { zar pelo seu dinheiro, pagar contas, lidar com o } \\
\text { proprietário ou inquilino, lidar com companhias } \\
\text { prestadoras de serviços ou serviços públicos. }\end{array}$ & $\begin{array}{l}\text { Foram incluídos exemplos de situações que } \\
\text { enfatizam o ato de administrar o orçamento, } \\
\text { como por exemplo: “saber quanto ganha, } \\
\text { "como está sua conta bancária”, visto que } \\
\text { a questão foi interpretada como "não ter } \\
\text { dinheiro”. }\end{array}$ \\
\hline D10 & $\begin{array}{l}\text { Take (taking) part in a regular fitness } \\
\text { program. This may include walking for } \\
\text { exercise, stationary biking, weight lifting } \\
\text { or exercises class. }\end{array}$ & $\begin{array}{l}\text { D10. Participa (participar) regularmente de } \\
\text { programa de atividades físicas. Por exemplo: } \\
\text { caminhada/corrida para exercício, bicicleta } \\
\text { ergométrica, musculação, academia, aulas de } \\
\text { ginástica. }\end{array}$ & $\begin{array}{l}\text { Para reforçar a diferenciação quanto ao } \\
\text { item D6, foram incluídos dois exemplos de } \\
\text { atividades de cunho eminentemente espor- } \\
\text { tivo: corrida e academia, que é praticado no } \\
\text { contexto brasileiro. }\end{array}$ \\
\hline D15 & $\begin{array}{l}\text { Take (taking) care of local errands. } \\
\text { This may include managing and taking } \\
\text { responsibility for shopping for food and } \\
\text { personal items, and going to the bank, } \\
\text { library, or dry cleaner. }\end{array}$ & $\begin{array}{l}\text { Cuida (cuidar) dos afazeres cotidianos. Isso } \\
\text { pode incluir: administrar e se responsabilizar } \\
\text { pela compra de alimentos e itens pessoais, ir ao } \\
\text { banco, banca de jornais ou casa lotérica. }\end{array}$ & $\begin{array}{l}\text { Os exemplos “biblioteca” e “lavanderia” } \\
\text { foram substituídos por “banca de jornal” e } \\
\text { “casa lotérica”, locais condizentes com o } \\
\text { nosso contexto. }\end{array}$ \\
\hline
\end{tabular}


TOLDRÁ, R. C. et al. Adaptação transcultural do Late-life. Rev. Ter. Ocup. Univ. São Paulo, v. 23, n. 1, p. 52-61, jan./abr. 2012.

Algumas situações e atividades foram substituídas, excluídas ou incluídas por serem mais representativas e habituais aos idosos brasileiros. Os exemplos a seguir ilustram essa equivalência. "Milha” foi substituída por "kilômetro" (item F4), "cordão de luz" por "cordão de descarga” (item F10); “porta externa” por “portão” (item F15); “celofane” por "pacote de salgadinhos ou biscoitos” (item F16); "fasteners” por "zíperes, botões, velcro, cinto e suspensório” (item F3).
As modificações implementadas durante o processo de adaptação transcultural consideraram a clareza, pertinência e a equivalência do instrumento ao nosso contexto. Na aplicação da $2^{a}$ versão, a equivalência foi testada novamente, até que nenhum item fosse considerado não aplicável por mais de $10 \%$ dos participantes, gerando a versão final do LLFDI em Português Brasileiro, agora denominada LLFDI-Br.

\section{ANEXO 1 - LLFDI-Br - INSTRUMENTO DE AVALIAÇÃO DA FUNÇÃO E DA INCAPACIDADE PARA A POPULAÇÃO IDOSA}

\section{PERGUNTAS SOBRE INCAPACIDADE}

\begin{tabular}{|c|c|c|c|c|c|c|c|c|c|c|}
\hline D1. Mantém (manter) contato com outras pessoas através de cartas ou telefonemas ou email. & 5 & 4 & 3 & 2 & 1 & 5 & 4 & 3 & 2 & 1 \\
\hline D2. Faz (fazer) visitas à casa de amigos e familiares. & 5 & 4 & 3 & 2 & 1 & 5 & 4 & 3 & 2 & 1 \\
\hline $\begin{array}{l}\text { D3. Cuida (cuidar) ou presta (prestar) assistência a outras pessoas. Por exemplo: prover cuida- } \\
\text { dos pessoais, transporte, fazer favores para membros da família ou amigos. }\end{array}$ & 5 & 4 & 3 & 2 & 1 & 5 & 4 & 3 & 2 & 1 \\
\hline $\begin{array}{l}\text { D4. Toma (tomar) conta da sua casa. Isso inclui administrar e se responsabilizar pelo cuidado } \\
\text { da casa: lavar a roupa, limpar a casa e fazer pequenos reparos. }\end{array}$ & 5 & 4 & 3 & 2 & 1 & 5 & 4 & 3 & 2 & 1 \\
\hline D5. Assume (assumir) trabalhos voluntários fora de casa. & 5 & 4 & 3 & 2 & 1 & 5 & 4 & 3 & 2 & 1 \\
\hline $\begin{array}{l}\text { D6. Faz (fazer) atividades de lazer ativo. Por exemplo: boliche, bocha, futebol/pelada, andar, } \\
\text { passear com cachorro, caminhada, dançar. }\end{array}$ & 5 & 4 & 3 & 2 & 1 & 5 & 4 & 3 & 2 & 1 \\
\hline $\begin{array}{l}\text { D7. Cuida (cuidar) da administração da casa e das finanças. Por exemplo: saber quanto ganha, } \\
\text { como está sua conta bancária, se responsabilizar pelo seu dinheiro, pagar contas, lidar com o } \\
\text { proprietário ou inquilino, lidar com companhias prestadoras de serviços ou serviços públicos. }\end{array}$ & 5 & 4 & 3 & 2 & 1 & 5 & 4 & 3 & 2 & 1 \\
\hline $\begin{array}{l}\text { D8. Cuida (cuidar) da sua própria saúde. Por exemplo: administrar medicação diária, seguir } \\
\text { uma dieta especial, agendar consultas médicas. }\end{array}$ & 5 & 4 & 3 & 2 & 1 & 5 & 4 & 3 & 2 & 1 \\
\hline D9. $\left(^{*}\right)$ Pensando no último ano, ...viaja (viajar) para fora da cidade por pelo menos uma noite. & 5 & 4 & 3 & 2 & 1 & 5 & 4 & 3 & 2 & 1 \\
\hline $\begin{array}{l}\text { D10. Participa (participar) regularmente de programa de atividades físicas. Por exemplo: cami- } \\
\text { nhada/corrida para exercício, bicicleta ergométrica, musculação, academia, aulas de ginástica. }\end{array}$ & 5 & 4 & 3 & 2 & 1 & 5 & 4 & 3 & 2 & 1 \\
\hline D11. Convida (convidar) pessoas para vir à sua casa para uma refeição ou lazer. & 5 & 4 & 3 & 2 & 1 & 5 & 4 & 3 & 2 & 1 \\
\hline D12. Sai (sair) com outras pessoas para lugares públicos como restaurantes ou cinema. & 5 & 4 & 3 & 2 & 1 & 5 & 4 & 3 & 2 & 1 \\
\hline D13. Faz (fazer) seus cuidados pessoais. Isso inclui: o banho, vestuário e uso do vaso sanitário. & 5 & 4 & 3 & 2 & 1 & 5 & 4 & 3 & 2 & 1 \\
\hline $\begin{array}{l}\text { D14. Participa (participar) de atividades sociais. Por exemplo: ir a clubes, centros de convivên- } \\
\text { cia para idosos, grupos comunitários ou religiosos e jogos de carta/tabuleiro. }\end{array}$ & 5 & 4 & 3 & 2 & 1 & 5 & 4 & 3 & 2 & 1 \\
\hline $\begin{array}{l}\text { D15. Cuida (cuidar) dos afazeres cotidianos. Por exemplo: administrar e se responsabilizar } \\
\text { pela compra de alimentos e itens pessoais, ir ao banco, banca de jornais ou casa lotérica. }\end{array}$ & 5 & 4 & 3 & 2 & 1 & 5 & 4 & 3 & 2 & 1 \\
\hline $\begin{array}{l}\text { D16. Prepara (preparar) suas próprias refeições. Isso inclui: planejar, cozinhar, servir, deixar } \\
\text { tudo em ordem. }\end{array}$ & 5 & 4 & 3 & 2 & 1 & 5 & 4 & 3 & 2 & 1 \\
\hline
\end{tabular}


TOLDRÁ, R. C. et al. Adaptação transcultural do Late-life. Rev. Ter. Ocup. Univ. São Paulo, v. 23, n. 1, p. 52-61, jan./abr. 2012.

ANEXO 2 - LLFDI-Br - INSTRUMENTO DE AVALIAÇÃO DA FUNÇÃO E DA INCAPACIDADE PARA A POPULAÇÃO IDOSA

\section{PERGUNTAS SOBRE FUNCIONALIDADE}

\begin{tabular}{|c|c|c|c|c|c|}
\hline $\begin{array}{l}\text { Quanta dificuldade o(a) Sr.(a) tem para...? } \\
\text { Considere que isso é sem a ajuda de uma pessoa e sem o uso de uma bengala, andador, cadeira de rodas (manual/moto- } \\
\text { rizada) ou carrinho motorizado. }\end{array}$ & 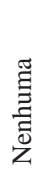 & $\begin{array}{l}\tilde{U} \\
\tilde{0} \\
\end{array}$ & 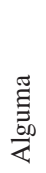 & 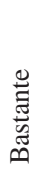 & 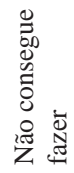 \\
\hline F1. Abrir a tampa de rosca de um pote que nunca foi aberto antes, sem auxílio de qualquer equipamento. & 5 & 4 & 3 & 2 & 1 \\
\hline F2. Subir e descer um lance de escadas em um espaço interno, usando um corrimão. & 5 & 4 & 3 & 2 & 1 \\
\hline F3. Colocar e tirar calças compridas (incluindo lidar com os zíperes, botões, velcro, cintos, suspensórios etc). & 5 & 4 & 3 & 2 & 1 \\
\hline F4. Correr $1 \mathrm{~km}$ ou mais. & 5 & 4 & 3 & 2 & 1 \\
\hline F5. Usar utensílios para preparar refeições (por exemplo, abridor de latas, descascador de batatas ou uma faca afiada). & 5 & 4 & 3 & 2 & 1 \\
\hline F6. Segurar um copo de água cheio com uma mão. & 5 & 4 & 3 & 2 & 1 \\
\hline F7. Andar 2 kms descansando quando necessário. & 5 & 4 & 3 & 2 & 1 \\
\hline F8. Subir e descer um lance de escadas sem corrimão ao ar livre. & 5 & 4 & 3 & 2 & 1 \\
\hline F9. Correr pequenas distâncias, como para pegar um ônibus. & 5 & 4 & 3 & 2 & 1 \\
\hline F10. Levantar e esticar o braço acima da cabeça, como para puxar o cordão da descarga estando em pé. & 5 & 4 & 3 & 2 & 1 \\
\hline F11. Sentar e levantar de um sofá baixo e macio. & 5 & 4 & 3 & 2 & 1 \\
\hline F12. Colocar e tirar um casaco ou jaqueta. & 5 & 4 & 3 & 2 & 1 \\
\hline F13. Alcançar as costas na altura da cintura, como se fosse passar o cinto por um passante da calça. & 5 & 4 & 3 & 2 & 1 \\
\hline F14. Subir e descer da calçada. & 5 & 4 & 3 & 2 & 1 \\
\hline F15. Abrir uma porta pesada (ex: um portão). & 5 & 4 & 3 & 2 & 1 \\
\hline F16. Abrir um pacote de salgadinhos ou biscoito usando as mãos. & 5 & 4 & 3 & 2 & 1 \\
\hline F17. Servir líquido de uma jarra grande. & 5 & 4 & 3 & 2 & 1 \\
\hline F18. Entrar e sair de um carro ou táxi (médio). & 5 & 4 & 3 & 2 & 1 \\
\hline F19. Fazer caminhadas por alguns quilômetros em superfícies irregulares, incluindo subidas. & 5 & 4 & 3 & 2 & 1 \\
\hline F20. Subir e descer 3 lances de escada em um espaço interno, usando um corrimão. & 5 & 4 & 3 & 2 & 1 \\
\hline F21. Levantar e tirar uma cadeira de cozinha para limpar o chão. & 5 & 4 & 3 & 2 & 1 \\
\hline F22. Usar um banquinho ou escadinha para alcançar um armário alto. & 5 & 4 & 3 & 2 & 1 \\
\hline F23. Arrumar a cama, incluindo esticar e prender os lençóis debaixo do colchão. & 5 & 4 & 3 & 2 & 1 \\
\hline F24. Subir um lance de escadas estando com as mãos ocupadas (por exemplo, carregando um cesto de roupas). & 5 & 4 & 3 & 2 & 1 \\
\hline F25. Estando em pé, abaixar-se para pegar uma peça de roupa no chão. & 5 & 4 & 3 & 2 & 1 \\
\hline F26. Andar pela casa, considerando a largura das portas, móveis, obstáculos, tapetes e diferentes tipos de piso. & 5 & 4 & 3 & 2 & 1 \\
\hline F27. Levantar-sedo chão a partir da posição deitada. & 5 & 4 & 3 & 2 & 1 \\
\hline F28. Em pé diante da pia, lavar pratos, panelas e utensílios com as mãos. & 5 & 4 & 3 & 2 & 1 \\
\hline F29. Andar vários quarteirões. & 5 & 4 & 3 & 2 & 1 \\
\hline F30. Caminhar rapidamente por um quilômetro sem parar para descansar. & 5 & 4 & 3 & 2 & 1 \\
\hline F31. Subir e descer do ônibus. & 5 & 4 & 3 & 2 & 1 \\
\hline F32. Caminhar ao ar livre em superfícies escorregadias. & 5 & 4 & 3 & 2 & 1 \\
\hline
\end{tabular}

Constatou-se que o uso de procedimentos recomendados pela literatura (BEATON et al., 2007; GUILLEMIN et al., 2003; REICHENHEIM; MORAES, 2007) foi fundamental para o processo de adaptação do instrumento.

Identificam-se como limitação do estudo, a não participação de sujeitos que utilizavam meios auxiliares de locomoção, o que poderia eventualmente revelar percepções, em relação à função e à incapacidade, especificas dessa população. No entanto, considera-se que esse fator não tenha comprometido o processo de produção da versão adaptada para o português brasileiro, uma vez que este se baseou na compreensão das questões do instrumento e não na forma como os sujeitos realizam suas atividades.

\section{CONSIDERAÇÕES FINAIS}

Acredita-se que o processo de adaptação transcultural do LLFDI para o português brasileiro foi conduzido de forma criteriosa, a partir da incorporação a essa versão, de 
inúmeras alterações e sugestões produzidas ao longo de todo o processo.

O estudo produzido vem preencher uma lacuna para os profissionais de terapia ocupacional e áreas afins, dada a falta de instrumentos traduzidos e validados para a realidade brasileira, que avaliem a função e a incapacidade na realização das atividades da vida diária, especificamente com a população idosa.
Sugere-se que o Instrumento de Avaliação da Função e Incapacidade para a População Idosa - LLFDI-Br possa avaliar de forma abrangente a Função e a Incapacidade de idosos em nosso país e, captar mudanças nos diferentes aspectos referentes a esses componentes.

Para que se confirme sua utilidade e valor para uso pelo terapeuta ocupacional e por outros profissionais de áreas afins, deve ser submetido a futuros testes de validação.

TOLDRÁ, R. C.; SOUTO, A. C. F.; BATISTA, M. P. P.; ALMEIDA, M. H. M. Cross-cultural adaptation of Late-life Function and Disability Instrument into Brazilian Portuguese. Rev. Ter. Ocup. Univ. São Paulo, v. 23, n. 1, p. 52-61, jan./abr. 2012.

ABSTRACT: This paper presents the cross-cultural adaptation of the "Late-Life Function Disability Instrument - LLFDI" into Brazilian Portuguese. LLFDI is designed to assess the degree of functional independence of community dwelling people over 60 years old and to capture changes in functional level and the degree of disability. It comprises two components: Function and Disability. The Function component assesses the degree of difficulty in performing activities and the Disability component assesses two dimensions: the frequency of performing activities and the degree of limitations as perceived by the subjects. The adaptation process involved translation, back translation, review by the Expert Committee and pre-test conducted with 47 subjects. The resulting brazilian portuguese version "Instrumento de Avaliação da Função e da Incapacidade para a População Idosa” (LLFDI-Br) was fully understood and may constitute as an important assessment tool for this population. Further studies are required to evaluate its psychometric properties.

KEYWORDS: Aging; Aged; Evaluation/methods; Translation; Adaptation; Functioning; Disability; International Classification of Functioning, Disability and Health.

\section{REFERÊNCIAS}

ABIZANDA, et al. Validation of the Spanish Version of the Shortform Late-life Function and Disability Instrument. JAGS. v. 59, n. 5, p. 893-899, 2011.

BEATON, B.; et al. Recommendations for the Cross-cultural adaptation of the DASH \&Quick DASH Outcome Measures. Institute for Work \& Health, 2007. Disponível em: http://www. dash.iwh.on.ca/how-translate.

BRASIL. Ministério da Saúde. Portaria 1395/GM. Política de Saúde do Idoso. Brasília, 1999.

BRASIL. Ministério da Saúde. Portaria n ${ }^{\circ}$ 2.528. Aprova a Política Nacional de Saúde da Pessoa Idosa. Brasília, 2006.

CICONELLI, R. M.; et al. Tradução para a língua portuguesa e validação do questionário genérico de avaliação de qualidade de vida SF-36 (Brasil SF-36). Rev. Bras. Reumatol. v. 39, n. 3, p.143-150, 1999.

DENKINGER, et al. Evaluation of the short form of the late-life function and disability instrument in geriatric inpatients: Validity, responsiveness and sensitivity to change. J. Am. Geriatr. Soc., v. 57, p. 309-314, 2009.
DIAS, E. G.; DUARTE, Y.A. O.; ALMEIDA, M. H. M.; LEBRÃO, M. L. Caracterização das atividades avançadas de vida diária (AAVDS): um estudo de revisão. Rev. Ter. Ocup. Univ. São Paulo, v. 22, n. 1, p. 45-51, 2011.

GUILLEMIN, F. G.; BOMBARDIER, C.; BEATON, D. Crosscultural adaptation of health-related quality of life measures: literature review and proposed guidelines. J. Clin. Epidemiol., v. 46, n. 12, p. 1417-1432, 1993.

JETTE, A. M.; et al. "Late Life Function and Disability Instrument: I. Development and Evaluation of the Disability Component”. J. Gerontol., v. 57A, n. 4, p. M209-M216, 2002.

JETTE, A. M.; HALEY, S. M.; KOOYOOMJIAN, J. T. Late-Life FDI Manual. Roybal Center for the Enhancement of Late-life Function, 2002. Disponível em: <http://sph.bu.edu/images/stories/ scfiles/hdri/LLFDI_Manual_2006_rev.pdf $>$.

LAWTON, M. P.; BRODY, E. M. Assessment of older people: self-maintaining and instrumental activities of daily living. Gerontologist, v. 9, p. 179-186, 1969.

LITVOC, J.; DERNTL, A. M. Capacidade funcional do idoso: 
TOLDRÁ, R. C. et al. Adaptação transcultural do Late-life. Rev. Ter. Ocup. Univ. São Paulo, v. 23, n. 1, p. 52-61, jan./abr. 2012.

significado e aplicações. In: CIANCIARULLO, T. I.; GUALDA, D. M. R.; SILVA, G. T. R.; CUNHA, I. C. K. O. Saúde na família e na comunidade. São Paulo: Robe editorial, 2002. p. 268-319.

MANSUR, L. L.; CARVALHO, C. R. F.; SCHOCHAT, E.; TANAKA, C.; LANCMAN, S.; ALMEIDA, M. H. M.; TOLDRÁ, R. C. "Envelhecer mantendo funções: idosos no ano de 2020," aprovado pelo edital MCT/CNPq/CT-Saúde $n^{\circ}$ 058/2009.

McAULEY, E.; KONOPACK, J. F.; MOTL, R. W.; ROSENGREN, K.; MORRIS, K. "Mesuring Disability and Function in Older Women: Psychometric Properties of the Late-life Function and Disability Instrument”. J. Gerontol., 2005. v. 60A, n. 7, p. 901909, 2005.

MELLO, M. A. F.; MANCINI, M. C. Avaliação das atividades de vida diária e controle domiciliary. In: CAVALCANTI, A.; GALVÃO C. Terapia ocupacional: fundamentação e prática. Rio de Janeiro: Guanabara Koogan, 2007. p. 49-54.

MELZER, et al. Relationship between self-reported function and disability and balance performances measures in the elderly. $J$. Rehabil. Res. Dev. v. 44, p. 685-692, 2007.
PAIXÃO Jr, C. M.; REICHENHEIM, M. E. Uma revisão sobre instrumentos de avaliação do estado funcional do idoso. Cad. Saúde Pública, Rio de Janeiro, v. 21, n. 1, p. 7-19, 2005.

REICHENHEIM, M. E.; MORAES, C. L. Operacionalização de Adaptação Transcultural de Instrumentos de Aferição usados em Epidemiologia. Rev. Saúde Pública, v. 41, n. 4, p. 665-677, 2007.

SAYERS, et al. "Validation of the Late-Life Function and Disability Instrument”. J. Am. Geriatr. Soc., v. 52, n. 9, p. 15541559, 2004.

SHIN, et al. A study on physical symptom, activity of daily living and health-related quality of life (HRQoL) in the communitydwelling older adults. J. Korean Acad. Nurs., v. 38, p. 437-444, 2008.

WONG, L. L. R.; CARVALHO, J. A. "O rápido processo de envelhecimento populacional do Brasil: sérios desafios para as políticas públicas”. Rev. Bras. Estudos Populac., São Paulo, v. 23, n. 1, p. 5-26, 2006. 